\title{
Fast sampling control of a class of differential linear repetitive processes
}

\author{
S E Benton ${ }^{1}$, E Rogers $^{1 *}$ and D H Owens ${ }^{2}$ \\ ${ }^{1}$ Department of Electronics and Computer Science, University of Southampton, UK \\ ${ }^{2}$ Department of Automatic Control and Systems Engineering, University of Sheffield, UK
}

\begin{abstract}
Repetitive processes are a distinct class of two-dimensional linear systems of practical and theoretical interest. Most of the available control theory for them is for the case of linear dynamics and focuses on systems theoretic properties such as stability and controllability/observability. This paper uses an extension of standard, or one-dimensional, feedback control schemes to control a physically relevant subclass of these processes.
\end{abstract}

Keywords: repetitive processes, control theory, fast sampling control, feedback

\section{NOTATION}

G(z) plant transfer function matrix

$h \quad$ sampling interval

$k \quad$ pass index $\geqslant 0$

$\mathbf{K}(z) \quad$ forward path controller transfer

function matrix

$r(\cdot) \quad$ spectral radius

$t, p \quad$ independent temporal or spatial variables

$\boldsymbol{u}_{k}(t), \boldsymbol{u}_{k}(p) \quad$ pass control input vector

$\boldsymbol{x}_{k}(t), \boldsymbol{x}_{k}(p) \quad$ pass state vector

$\boldsymbol{y}_{k}(t), \boldsymbol{y}_{k}(p) \quad$ pass profile vector

$\alpha \quad$ pass length $<\infty$

$\|\cdot\| \quad$ norm, induced operator norm

\section{INTRODUCTION}

Repetitive (also termed multipass in the early literature) processes are characterized by a series of sweeps, termed passes, through a set of dynamics defined over a fixed finite duration known as the pass length. On each pass an output, termed the pass profile, is produced which acts as a forcing function on, and hence contributes to, the dynamics of the next pass profile. Industrial examples

The MS was received on 5 December 2000 and was accepted after revision for publication on 24 July 2001.

* Corresponding author: Department of Electronics and Computer Science, University of Southampton, Highfield, Southampton SO17 1BJ, UK. include long-wall coal cutting and metal rolling (see, for example, references [1] and [2] and the relevant cited references for a detailed treatment of these and other physical examples), and algorithmic examples include classes of iterative learning control schemes [3] and solution algorithms for non-linear dynamic optimal control algorithms based on the maximum principle [4]. The designation 'algorithmic' here denotes the fact that in such applications adopting a repetitive process setting for analysis has distinct/unique advantages over alternative approaches.

The unique control problem for these processes is that the output sequence of pass profiles can contain oscillations that increase in amplitude in the pass-to-pass direction. Such behaviour is easily generated in simulation studies and in experiments on scaled models of physical examples such as long-wall coal cutting and cannot, in almost all cases, be removed by the application of a standard, termed one-dimensional here, control action. This is precisely because such an approach essentially ignores their inherent two-dimensional linear systems structure, i.e. information propagation in two separate directions - along a given pass and from pass to pass respectively. In particular, repetitive processes are a class of two-dimensional systems whose distinguishing feature (from other classes of such systems) is that information propagation in one of the two separate (or distinct) directions only takes place over a finite and fixed duration - the pass length.

A rigorous stability theory for linear constant pass length processes has been developed $[\mathbf{5}, \mathbf{6}]$. This theory is based on an abstract model in a Banach space setting which includes all such processes as special cases. Also, 
the results of applying this abstract theory to a range of subclasses of practical interest are known. These include so-called differential and discrete linear repetitive processes which are the subject of this paper.

Differential linear repetitive processes have strong structural links with one-dimensional differential linear systems. For example, the stability conditions in this case can be tested by direct application of well-known onedimensional linear systems tests. This raises the natural question of what exactly can be achieved by the use of one-dimensional feedback control schemes in this context, e.g. is it possible to use a one-dimensional unity negative feedback control scheme to stabilize these processes. The results of this paper show that this problem has a solution in one case of practical interest but only when feedback control action on the current pass is augmented by well-defined feedforward action from the previous pass.

\section{BACKGROUND}

The essential unique characteristic of a repetitive process can be illustrated by considering machining operations where the material or workpiece involved is processed by a series of sweeps, termed passes, of a processing tool. Assuming that the pass length $\alpha<+\infty$ is constant, the output vector, or pass profile $\boldsymbol{y}_{k}(t), 0 \leqslant t \leqslant \alpha$, generated on pass $k$ acts as a forcing function on, and hence contributes to, the dynamics of the new pass profile $\boldsymbol{y}_{k+1}(t)$, $0 \leqslant t \leqslant \alpha, k \geqslant 0$. This explicit interaction between successive pass profiles is the source of the unique control problem for these processes in that the sequence of pass profiles generated $\left\{\boldsymbol{y}_{k}\right\}_{k \geqslant 1}$ can contain oscillations that increase in amplitude in the pass-to-pass (i.e. $k$ ) direction.

In the case of long-wall cutting (see reference [1]), the unique control problem appears as severe undulations in the newly cut floor profile [caused by the machine weight (up to $5 \mathrm{t}$ in some cases) as it proceeds along the coal face resting on the floor profile cut during the previous pass] which means that cutting operations (i.e. productive work) must be suspended to enable their manual removal. This problem is one of the key factors behind the 'stop/start' cutting pattern in a typical working cycle in a coal mine. Also, as with all repetitive processes, this problem cannot be removed by direct application of a one-dimensional control action precisely because such an approach essentially ignores their finite pass length repeatable nature and the effects of resetting the initial conditions prior to starting the next pass. Instead, the control of these processes must be based on abstract model based stability theory $[\mathbf{5}, \mathbf{6}]$.

This paper considers the case of differential linear repetitive processes whose state-space model over
$0 \leqslant t \leqslant \alpha$ and $k \geqslant 0$ has the form

$$
\begin{aligned}
& \dot{\boldsymbol{x}}_{k+1}(t)=\mathbf{A} \boldsymbol{x}_{k+1}(t)+\mathbf{B} \boldsymbol{u}_{k^{+}}{ }_{1}(t)+\mathbf{B}_{0} \boldsymbol{y}_{k}(t) \\
& \boldsymbol{y}_{k+1}(t)=\mathbf{C} \boldsymbol{x}_{k+1}(t)+\mathbf{D}_{0} \boldsymbol{y}_{k}(t)
\end{aligned}
$$

Here, on pass $k, \boldsymbol{x}_{k}(t)$ is the $n \times 1$ state vector, $\boldsymbol{y}_{k}(t)$ is the $m \times 1$ vector pass profile and $\boldsymbol{u}_{k}(t)$ is the $l \times 1$ vector of control inputs. To complete the process description it is necessary to specify the initial conditions, i.e. the pass state initial vector sequence and the initial pass profile. These are also termed the boundary conditions, and the simplest possible form of them is assumed in this work, i.e.

$$
\begin{array}{ll}
\boldsymbol{x}_{k^{+}}(0)=\boldsymbol{d}_{k^{+1}}, & k \geqslant 0 \\
\boldsymbol{y}_{0}(t)=\boldsymbol{y}(t), & 0 \leqslant t \leqslant \alpha
\end{array}
$$

where $\boldsymbol{d}_{k+1}$ is a constant $n \times 1$ vector and the elements in the $m \times 1$ vector $\boldsymbol{y}(t)$ are known functions of $t$. Note also that the structure of the boundary conditions alone has a critical influence on the stability properties of a given example [7]. (Essentially, reference [7] shows that a stable example described by (1) and (2) can be made unstable by adding to $\boldsymbol{x}_{k+1}(0)$ a term that is an explicit function of points along the previous pass profile.)

The stability theory $[\mathbf{5 , 6}]$ is based on an abstract model in a Banach space setting which includes all processes with linear dynamics and a constant pass length as special cases. This theory consists of two separate concepts termed asymptotic stability and stability along the pass respectively. In effect, asymptotic stability demands that bounded input sequences produce bounded output sequences of pass profiles (in terms of the norm on the underlying function space) over the (finite and constant) pass length. Stability along the pass is the stronger concept that bounded input sequences produce bounded sequences of pass profiles independent of the pass length. Asymptotic stability over all possible pass lengths is a necessary condition for stability along the pass and, although applications do exist (see references [3] and [4]) where asymptotic stability is all that is required or achievable, it is stability along the pass that is required in the vast majority of cases.

The following result gives necessary and sufficient conditions for stability along the pass of processes described by (1) and (2).

\section{Theorem 1}

Suppose that the pair $\left\{\mathbf{A}, \mathbf{B}_{0}\right\}$ is controllable and the pair $\{\mathbf{C}, \mathbf{A}\}$ is observable. Then differential linear repetitive processes described by (1) and (2) are stable along the pass if, and only if:

(a) all eigenvalues of matrix $\mathbf{D}_{0}$ have a modulus strictly less than unity; 
(b) all eigenvalues of matrix A have strictly negative real parts;

(c) all eigenvalues of the transfer function matrix

$$
\mathbf{G}(s)=\mathbf{C}\left(s \mathbf{I}_{n}-\mathbf{A}\right)^{-1} \mathbf{B}_{0}+\mathbf{D}_{0}
$$

have a modulus strictly less than unity $\forall s=\iota \omega$, $\omega \geqslant 0$.

A key advantage of this set of conditions is that they can be tested by direct application of one-dimensional (differential and discrete as required) linear systems stability tests. Also, each of them has a well-defined physical interpretation. In particular, condition (a) in this result is the necessary and sufficient condition for asymptotic stability (asymptotic stability for all possible values of the pass length is a necessary condition for stability along the pass) and, in physical terms, demands that the sequence of pass initial responses $\left\{\boldsymbol{y}_{k}(0)\right\}_{k \geqslant 1}$ does not become unbounded in a well-defined sense as the pass number $k \rightarrow \infty$. Condition (b) governs the dynamics produced along a given pass but is only a necessary condition for stability along the pass (e.g. the example below). Finally, condition (c) can be interpreted physically as the requirement that each frequency component of the initial profile is attenuated from pass to pass [and not just the d.c. component as in condition (a) here].

The so-called limit profile, whose existence is guaranteed by asymptotic stability and hence stability along the pass, provides information on transient behaviour in the pass-to-pass (i.e. $k$ ) direction. Suppose, therefore, that a process described by (1) and (2) is asymptotically stable and the control input sequence applied $\left\{\boldsymbol{u}_{\mathrm{k}}\right\}_{k \geqslant 1}$ converges strongly (in the sense of the norm on the underlying function space) to $\boldsymbol{u}_{\infty}$. Then the resulting limit profile is formally defined in terms of the output sequence of pass profiles $\left\{\boldsymbol{y}_{\mathrm{k}}\right\}_{k \geqslant 1}$ as the strong limit

$$
\boldsymbol{y}_{\infty}:=\lim _{k \rightarrow+\infty} \boldsymbol{y}_{k}
$$

In state-space terms, the limit profile of a process described by (1) and (2) is described by the following one-dimensional differential linear systems state-space model over $0 \leqslant t \leqslant \alpha$ :

$$
\begin{aligned}
& \dot{\boldsymbol{x}}_{\infty}(t)=\left(\mathbf{A}+\mathbf{B}_{0}\left(\mathbf{I}_{m}-\mathbf{D}_{0}\right)^{-1} \mathbf{C}\right) \boldsymbol{x}_{\infty}(t)+\mathbf{B} \boldsymbol{u}_{\infty}(t) \\
& \boldsymbol{y}_{\infty}(t)=\left(\mathbf{I}_{m}-\mathbf{D}_{0}\right)^{-1} \mathbf{C} \boldsymbol{x}_{\infty}(t), \quad \boldsymbol{x}_{\infty}(0)=\boldsymbol{d}_{\infty}
\end{aligned}
$$

where $\boldsymbol{d}_{\infty}$ denotes the strong limit of the sequence $\left\{\boldsymbol{d}_{k}\right\}_{k \geqslant 1}$.

As noted above, asymptotic stability guarantees the existence of the limit profile for processes described by (1) and (2) which is simply a strictly proper onedimensional differential linear time-invariant system. This property does not, however, guarantee that the resulting limit profile has the most basic acceptable pass dynamics, i.e. stability as a one-dimensional linear system.
A simple example that demonstrates this key fact is the following process where $\beta>0$ is a real scalar:

$$
\begin{aligned}
& \dot{y}_{k^{+} 1}(t)=-\boldsymbol{y}_{k^{+} 1}(t)+\boldsymbol{u}_{k^{+} 1}(t)+(1+\beta) y_{k}(t) \\
& x_{k^{+} 1}(0)=0, \\
& \boldsymbol{y}_{k^{+} 1}(t)=\boldsymbol{x}_{k^{+} 1}(t), \quad 0 \leqslant t \leqslant \alpha
\end{aligned}
$$

which is asymptotically stable with limit profile

$$
\dot{\boldsymbol{y}}_{\infty}(t)=\beta_{\boldsymbol{y}_{\infty}}(t)+\boldsymbol{u}_{\infty}(t), \quad \boldsymbol{y}_{\infty}(0)=0, \quad 0 \leqslant t \leqslant \alpha
$$

Also, if $\boldsymbol{u}_{k+1}(t) \equiv 1$ and $\boldsymbol{y}_{0}(t)=\boldsymbol{y}(t) \equiv 0,0 \leqslant t \leqslant \alpha, k \geqslant 0$,

$$
\begin{array}{ll}
y_{1}(t)=1-\mathrm{e}^{-t}, & 0 \leqslant t \leqslant \alpha \\
y_{\infty}(t)=\beta^{-1}\left(\mathrm{e}^{\beta t}-1\right), & 0 \leqslant t \leqslant \alpha
\end{array}
$$

i.e. despite the fact that the first pass profile $\boldsymbol{y}_{1}(t)$ is a quite acceptable 'classical response' to the unit step command, the resulting limit profile has quite unacceptable dynamic characterisics. In particular, its pass dynamics grow exponentially and can be said to be "unstable along the pass' in an obvious intuitive sense. In terms of Theorem 1, it is condition (c) that does not hold if $\beta>0$ since $\mathbf{G}(s)=(1+\beta) /(s+1)$. Hence, stability along the pass requires that $\beta<0$.

\section{CONTROL AND PERFORMANCE SPECIFICATIONS}

The form of the limit profile for differential [and discrete (see references [5] and [6])] linear repetitive processes has led (see reference [2] for details) to the following general-purpose performance specification in addition to the obvious requirement of stability along the pass. Drive the output sequence of pass profiles $\left\{\boldsymbol{y}_{k}\right\}_{k \geqslant 1}$ to a limit profile $\boldsymbol{y}_{\infty}$ with 'acceptable' along the pass dynamics. 'Practical' convergence should occur in a 'reasonable' number of passes and, simultaneously, 'tolerable' errors on any pass $k$ should be guaranteed.

Many of these features will need interpretation in the context of the particular application under consideration. Further details on this central issue can be found in reference [2], but here the focus is on the use of fast sampling control, the precise meaning of which will be given later in the paper.

Consider the output of a process described by (1) and (2) at instant $t$ on pass $k$. Then the information in the following set is available to be used for output feedback control:

$$
\begin{aligned}
& \mathbf{Y}=\mathbf{Y}_{1} \cup \mathbf{Y}_{2} \\
& \mathbf{Y}_{1}=\left\{\boldsymbol{y}_{k}(\tau): 0 \leqslant \tau \leqslant t\right\} \\
& \mathbf{Y}_{2}=\left\{\boldsymbol{y}_{\hat{k}}(\tau): 0 \leqslant \tau \leqslant \alpha, 0 \leqslant \hat{k} \leqslant k-1\right\}
\end{aligned}
$$


Clearly, however, the most appealing schemes from an implementation standpoint will be those that explicitly use only information from pass $k$ since they have an obviously simpler structure (in terms, for example, of the information required to calculate the control signal at a given instant). The simplest of all the possible controllers that only use current pass information are so-called memoryless schemes which compute the control signal at $(k, t)$ using only information at this instant. All such schemes effectively ignore the previous pass dynamics and essentially regard them as disturbances that need to be rejected.

Such schemes have been extensively investigated in, for example, reference [2], and it has been concluded that (as may be expected intuitively) they are somewhat limited in terms of the subclasses of (1) and (2) (and the natural discrete counterpart) to which they can be successively applied. Also, if previous pass information is to be explicitly included in the controller(s) then there is, as yet, little known about the most effective way of using such information. This paper gives the first major results on this key question in terms of so-called memoryless dynamic unity negative feedback control schemes with repetitive minor loop compensation. Such schemes have the form

$$
\boldsymbol{u}_{k+1}(t)=\hat{\boldsymbol{y}}_{k+1}(t)-\mathbf{K}_{1} \boldsymbol{y}_{k}(t), \quad 0 \leqslant t \leqslant \alpha, \quad k \geqslant 0
$$

where $\hat{\boldsymbol{y}}_{k^{+}}(t)$ is the output of a one-dimensional linear time-invariant system defined by the state-space quadruple $\{\mathbf{F}, \mathbf{G}, \mathbf{H}, \mathbf{J}\}$ actuated by the current pass error vector $\boldsymbol{e}_{k^{+}}(t)$ which is defined as $\boldsymbol{e}_{k^{+}}(t)=$ $\boldsymbol{r}_{k+1}(t)-\boldsymbol{y}_{k^{+} 1}(t)$, where $\boldsymbol{r}_{k^{+}}(t)$ is an $m \times 1$ reference vector taken to represent the desired behaviour on pass $k+1$. The so-called previous pass controller $\mathbf{K}_{1}$ has (in this case) constant entries. In the remainder of this paper, new results are developed on the design of the digital form of control schemes with the structure of (10) to yield high-performance control of a subclass of processes described by (1) and (2) under fast sampling action (defined in the next section).

\section{FAST SAMPLING}

The natural discrete analogue of (1) is the so-called discrete repetitive process with the following state-space model over $0 \leqslant p \leqslant \alpha$ and $k \geqslant 0$ :

$$
\begin{aligned}
& \boldsymbol{x}_{k^{+} 1}(p+1)=\boldsymbol{\Phi}_{\boldsymbol{x}_{k+1}}(p)+\boldsymbol{\Delta}_{\boldsymbol{u}_{k+1}}(p)+\boldsymbol{\Delta}_{0} \boldsymbol{y}_{k}(p) \\
& \boldsymbol{y}_{k+1}(p)=\mathbf{C} \boldsymbol{x}_{k+1}(p)+\mathbf{D}_{0} \boldsymbol{y}_{k}(p)
\end{aligned}
$$

where the current pass state, pass profile and input vectors have the same dimensions as in (1). Also, the simplest possible form is again assumed for the boundary conditions, i.e. the discrete equivalents of the entries in
(2) in the following form:

$$
\begin{array}{ll}
\boldsymbol{x}_{k+1}(0)=\boldsymbol{d}_{k+1}, & k \geqslant 0 \\
\boldsymbol{y}_{0}(p)=\boldsymbol{y}(p), & 0 \leqslant p \leqslant \alpha
\end{array}
$$

where $\boldsymbol{d}_{k^{+}}$is an $n \times 1$ vector with constant entries and the elements in the $m \times 1$ vector $\boldsymbol{y}(p)$ are known functions of $p$. Necessary and sufficient conditions for stability along the pass of processes described by (11) and (12) are given by the following result.

\section{Theorem 2}

Suppose that the pair $\left\{\boldsymbol{\Phi}, \boldsymbol{\Delta}_{0}\right\}$ is controllable and the pair $\{\mathbf{C}, \boldsymbol{\Phi}\}$ is observable. Then discrete linear repetitive processes described by (11) and (12) are stable along the pass if, and only if:

(a) all eigenvalues of matrices $\mathbf{D}_{0}$ and $\boldsymbol{\Phi}$ have a modulus strictly less than unity;

(b) all eigenvalues of the transfer function matrix

$$
\mathbf{G}(z)=\mathbf{C}\left(z \mathbf{I}_{n}-\boldsymbol{\Phi}\right)^{-1} \boldsymbol{\Delta}_{0}+\mathbf{D}_{0}
$$

have a modulus strictly less than unity $\forall|z|=1$.

Note also that no loss of generality arises from setting $\mathbf{D}_{0}=0$ and $\boldsymbol{d}_{k+1}=0, k \geqslant 0$, for both differential and discrete processes in what follows in this paper.

In the remainder of this section, it will be shown how a model of the form (11) and (12) results from sampling a process of the form (1) and (2), and the achievable accuracy will be considered. To start with, first write (1) and (2) in the form

$$
\begin{array}{r}
\boldsymbol{y}_{k^{+}}(t)=\mathbf{C} \int_{0}^{t} \mathrm{e}^{\mathrm{A}(t-\tau)}\left[\mathbf{B}_{0} \boldsymbol{y}_{k}(\tau)+\mathbf{B} \boldsymbol{u}_{k^{+}}(\tau)\right] \mathrm{d} \tau, \\
0 \leqslant t \leqslant \alpha, k \geqslant 0
\end{array}
$$

Suppose also that this model is subject to synchronous sampling with sampling interval $h$ and a piecewise constant input vector, i.e.

$$
\boldsymbol{u}_{k+1}(t)=\boldsymbol{u}_{k+1}(p h):=\boldsymbol{u}_{k+1}^{p}
$$

where $p h \leqslant t<(p+1) h$ and $0 \leqslant p \leqslant \alpha / h$. Also, write $\boldsymbol{x}_{k+1}^{p}:=\boldsymbol{x}_{k+1}(p h), \boldsymbol{y}_{k+1}^{p}:=\boldsymbol{y}_{k^{+}}(p h), 0 \leqslant p \leqslant \alpha / h$. Then, at $t=p h$, (14) can be written for $k \geqslant 0$ as

$$
\boldsymbol{y}_{k+1}(p h)=\mathbf{C} \int_{0}^{p h} \mathrm{e}^{\mathrm{A}(p h-\tau)}\left[\mathbf{B}_{0} \boldsymbol{y}_{k}(\tau)+\mathbf{B} \boldsymbol{u}_{k+1}(\tau)\right] \mathrm{d} \tau
$$

and also the state equation in (1) takes the following form:

$$
\boldsymbol{x}_{k+1}(p h)=\mathrm{e}^{\mathrm{A} p h}\left\{\int_{0}^{p h} \mathrm{e}^{-\mathrm{A} \tau}\left[\mathbf{B}_{0} \boldsymbol{y}_{k}(\tau)+\mathbf{B} \boldsymbol{u}_{k+1}(\tau)\right] \mathrm{d} \tau\right\}
$$


At instant $t=(p+1) h$, the following holds for $k \geqslant 0$ :

$$
\begin{aligned}
& \boldsymbol{x}_{k^{+}+}((p+1) h)=\mathrm{e}^{\mathrm{A} h}\left\{\boldsymbol{x}_{k^{+}}(p h)\right. \\
& \left.+\mathrm{e}^{\mathrm{A} p h} \int_{p h}^{(p+1) h} \mathrm{e}^{\mathrm{A} h} \mathrm{e}^{-\mathrm{A} \tau}\left[\mathbf{B}_{0} \boldsymbol{y}_{k}(\tau)+\mathbf{B} \boldsymbol{u}_{k^{+}}(\tau)\right] \mathrm{d} \tau\right\}
\end{aligned}
$$

and each term in turn in this equation is now considered. In particular, since the current pass input vector is assumed to be piecewise continuous, i.e. $\boldsymbol{u}_{k^{+}}{ }_{1}(t) \equiv$ $\boldsymbol{u}_{k+1}(p h)$ on $[p h,(p+1) h)$, it is possible to write, for $0 \leqslant p \leqslant \alpha / h$ and $k \geqslant 0$,

$$
\begin{aligned}
\mathrm{e}^{\mathrm{A} h} \mathrm{e}^{\mathrm{A} p h} \int_{p h}^{(p+1) h} \mathrm{e}^{-\mathrm{A} \tau} \mathbf{B} \boldsymbol{u}_{k+1}(\tau) \mathrm{d} \tau \\
=\mathrm{e}^{\mathrm{A} h} \mathrm{e}^{\mathrm{A} p h} \mathrm{e}^{-\mathrm{A} p h} \int_{0}^{h} \mathrm{e}^{-\mathrm{A} \tau} \mathbf{B} \mathrm{d} \tau \boldsymbol{u}_{k+1}(p h) \\
=\mathrm{e}^{\mathrm{A} h} \int_{0}^{h} \mathrm{e}^{-\mathrm{A} \tau} \mathbf{B} \mathrm{d} \tau \boldsymbol{u}_{k+1}^{p}
\end{aligned}
$$

Also, under the assumption that $h \rightarrow 0$, which is termed fast sampling, $\boldsymbol{y}_{k}(t)$ over the interval $[p h,(p+1) h)$ can be approximated by $\boldsymbol{y}_{k}(p h), 0 \leqslant p \leqslant \alpha / h, k \geqslant 0$. The accuracy of this approximation improves with decreasing $h$ and on $[p h,(p+1) h)$,

$$
\lim _{h \rightarrow 0} y_{k}(\tau)=y_{k}(p h)
$$

which is equivalent to the assumption that the previous pass profile is piecewise continuous. Hence with this assumption, the term involving $\boldsymbol{y}_{\boldsymbol{k}}(t)$ in equation (18) can be written as

$$
\mathrm{e}^{\mathrm{A} h} \mathrm{e}^{\mathrm{A} p h} \int_{p h}^{\left(p^{+} 1\right) h} \mathrm{e}^{-\mathrm{A} \tau} \mathbf{B}_{0} \boldsymbol{y}_{k}(\tau) \mathrm{d} \tau=\mathrm{e}^{\mathrm{A} h} \int_{0}^{h} \mathrm{e}^{-\mathrm{A} \tau} \mathbf{B}_{0} \mathrm{~d} \tau \boldsymbol{y}_{k}^{p}
$$

It is now also possible to write

$$
x_{k+1}^{p+1}=\mathrm{e}^{\mathrm{A} h} \boldsymbol{x}_{k+1}^{p}+\mathrm{e}^{\mathrm{A} h} \int_{0}^{h} \mathrm{e}^{-\mathrm{A} \tau}\left(\mathbf{B} \mathrm{d} \tau \boldsymbol{u}_{k+1}^{p}+\mathbf{B}_{0} \mathrm{~d} \tau \boldsymbol{y}_{k}^{p}\right)
$$

Then it follows from comparison with process (11) that

$$
\begin{aligned}
& \boldsymbol{\Phi}=\mathrm{e}^{\mathrm{A} h} \\
& \boldsymbol{\Delta}=\boldsymbol{\Phi} \int_{0}^{h} \mathrm{e}^{-\mathrm{A} \tau} \mathbf{B} \mathrm{d} \tau \\
& \boldsymbol{\Delta}_{0}=\boldsymbol{\Phi} \int_{0}^{h} \mathrm{e}^{-\mathrm{A} \tau} \mathbf{B}_{0} \mathrm{~d} \tau
\end{aligned}
$$

and also that the matrices defining the output (pass profile vector) updating are unchanged by the sampling operation.

Now it will be shown that the discrete linear negative process (11) and (12) obtained via the above synchron- ous sampling scheme becomes a differential linear repetitive process of form (1) and (2) in the limit as $h \rightarrow 0$. To start with, first note that from equation (22) it is possible to write

$$
\begin{aligned}
\lim _{h \rightarrow 0}\left(\boldsymbol{x}_{k^{+}}^{p^{+}} 1\right. & \left.-\boldsymbol{x}_{k^{+}}^{p}\right) \\
=\lim _{h \rightarrow 0^{+}} & {\left[\left(\mathrm{e}^{\mathrm{A} h}-\mathbf{I}_{h}\right) \boldsymbol{x}_{k^{+} 1}^{p}+\mathrm{e}^{\mathrm{A} h} \int_{0}^{h} \mathrm{e}^{-\mathrm{A} \tau} \mathbf{B} \mathrm{d} \tau \boldsymbol{u}_{k+1}^{p}\right.} \\
& \left.+\mathrm{e}^{\mathrm{A} h} \int_{0}^{h} \mathrm{e}^{-\mathrm{A} \tau} \mathbf{B}_{0} \mathrm{~d} \tau \boldsymbol{y}_{k}^{p}\right]
\end{aligned}
$$

Each term in this last equation will now be individually considered.

Consider firstly the term involving $\boldsymbol{x}_{k+1}^{p}$. Then, using the power series expansion for $\mathrm{e}^{\mathrm{A} h}$, it is possible to write

$$
\begin{aligned}
\lim _{h \rightarrow 0} \frac{1}{h}\left(\mathrm{e}^{\mathrm{A} h}-\mathbf{I}_{n}\right) \boldsymbol{x}_{k+1}^{p} & =\lim _{h \rightarrow 0}\left[\mathbf{I}_{n}+\mathbf{A} h+\mathbf{O}\left(h^{2}\right)-\mathbf{I}_{n}\right] \boldsymbol{x}_{k+1}^{p} \\
& =\mathbf{A} \boldsymbol{x}_{k+1}^{p}
\end{aligned}
$$

where $\mathbf{O}\left(h^{2}\right)$ represents terms in $h^{2}$ and higher powers. In the case of the term involving $\boldsymbol{u}_{k+1}^{p}$, it is possible to write

$$
\begin{aligned}
\lim _{h \rightarrow 0} \frac{1}{h}\left(\mathrm{e}^{\mathrm{A} h} \int_{0}^{h} \mathrm{e}^{-\mathrm{A} \tau} \mathbf{B} \mathrm{d} \tau \boldsymbol{u}_{k+1}^{p}\right) \\
=\lim _{h \rightarrow 0} \frac{1}{h}\left\{\left[\mathbf{I}_{n}+\mathbf{A} h+\mathbf{O}\left(h^{2}\right)\right]\right. \\
\left.\quad \times \int_{0}^{h}\left[\mathbf{I}_{n}-\mathbf{A} \tau+\mathbf{O}\left(\tau^{2}\right)\right] \mathbf{B} \mathrm{d} \tau \boldsymbol{u}_{k+1}^{p}\right\} \\
=\lim _{h \rightarrow 0} \frac{1}{h}\left\{\left[\mathbf{I}_{n}+\mathbf{A} h+\mathbf{O}\left(h^{2}\right)\right]\left[h+\mathbf{O}\left(h^{2}\right)\right] \boldsymbol{u}_{k+1}^{p}\right\} \\
=\mathbf{B} \boldsymbol{u}_{k+1}^{p}
\end{aligned}
$$

In the case of the term involving $\boldsymbol{y}_{k}^{p}$, a similar analysis to that of equation (26) shows that, as $h \rightarrow 0$, this term can be replaced by $\mathbf{B}_{0} \boldsymbol{y}_{k}^{p}$. Also, as $h \rightarrow 0, \boldsymbol{u}_{k+1}^{p}$ and $\boldsymbol{y}_{k}^{p}$ become continuous variables.

Combining the above results, and noting that, in the limit,

$$
\lim _{h \rightarrow 0} \frac{1}{h}\left(\boldsymbol{x}_{k^{+}}^{p_{1}^{+}}-\boldsymbol{x}_{k^{+}}^{p}\right)=\dot{\boldsymbol{x}}_{k^{+}}^{p}
$$

it is possible to write

$$
\dot{\boldsymbol{x}}_{k+1}^{p}=\mathbf{A} \boldsymbol{x}_{k+1}^{p}+\mathbf{B} \boldsymbol{u}_{k+1}^{p}+\mathbf{B}_{0} \boldsymbol{y}_{k}^{p}
$$

which, as $h \rightarrow 0$, approaches

$$
\dot{\boldsymbol{x}}_{k+1}(t)=\mathbf{A} \boldsymbol{x}_{k^{+}}(t)+\mathbf{B u}_{k^{+} 1}(t)+\mathbf{B}_{0} \boldsymbol{y}_{k}(t)
$$

This last equation, when combined with the fact that both the output equation and the boundary conditions in this case are static, is just the differential linear repetitive process state-space model of (1) and (2). 


\section{DISCRETE MULTIVARIATE FIRST-ORDER LAG ANALYSIS AND DESIGN}

Consider first the case of a controllable and observable one-dimensional discrete linear system with $m$ inputs and $m$ outputs whose state-space model is defined by the state-space triple $\{\boldsymbol{\Phi}, \boldsymbol{\Delta}, \mathbf{C}\}$ with inverse transfer function matrix

$$
\mathbf{G}^{-1}(z)=(z-1) \hat{\mathbf{B}}_{0}+\hat{\mathbf{B}}_{1}
$$

where $\hat{\mathbf{B}}_{0}$ and $\hat{\mathbf{B}}_{1}$ are real $m \times m$ matrices and $\operatorname{det}\left(\hat{\mathbf{B}}_{0}\right) \neq 0$. An equivalent definition is an $m$-input $m$-output discrete time model with state dimension equal to the output vector dimension and $\operatorname{det}(\mathbf{C} \boldsymbol{\Delta}) \neq 0$, i.e. the transfer function matrix in this case has minimal state space realization defined by

$$
\boldsymbol{\Phi}=\mathbf{I}_{m}-\hat{\mathbf{B}}_{0}^{-1} \hat{\mathbf{B}}_{1}, \quad \boldsymbol{\Delta}=\hat{\mathbf{B}}_{0}^{-1}, \quad \mathbf{C}=\mathbf{I}_{m}
$$

A one-dimensional discrete linear system with this structure is termed a discrete multivariable lag (by analogy with the classical single-input single-output case), and a 'rich' control theory for such processes exists [8].

In the case of discrete linear repetitive processes, the natural generalization of the multivariable first-order lag structure over $0 \leqslant p \leqslant \alpha$ and $k \geqslant 0$ is

$$
\begin{aligned}
& \tilde{\boldsymbol{x}}_{k+1}(p+1)=\left(\mathbf{I}_{m}-\hat{\mathbf{B}}_{0}^{-1} \hat{\mathbf{B}}_{1}\right) \tilde{\boldsymbol{x}}_{k+1}(p)+\hat{\mathbf{B}}_{0}^{-1} \boldsymbol{u}_{k+1}(p) \\
&+\hat{\boldsymbol{\Delta}}_{0} \boldsymbol{y}_{k}(p) \\
& \boldsymbol{y}_{k+1}(p)=\tilde{\boldsymbol{x}}_{k+1}(p)
\end{aligned}
$$

which can, in fact, be obtained from (11) (with $\mathbf{D}_{0}=0$ ) by using a simple transformation of the current pass state vector. Consider also now the discrete equivalent of the control scheme of (10) over $0 \leqslant p \leqslant \alpha$ and $k \geqslant 0$, i.e.

$$
\boldsymbol{u}_{k+1}(p)=\hat{\boldsymbol{y}}_{k^{+}}(p)-\mathbf{K}_{1} \boldsymbol{y}_{k}(p)
$$

and set

$$
\mathbf{K}_{1}=\hat{\mathbf{B}}_{0} \hat{\boldsymbol{\Delta}}_{0}
$$

Then it follows that the resulting closed-loop system in this case can be written in $z$ transform terms (see reference [2] for the details of how to avoid potential problems caused by the fact that the pass length is finite) as

$$
\mathbf{Y}_{k+1}(z)=\mathbf{H}(z) \mathbf{R}_{k+1}(z)
$$

where

$$
\begin{aligned}
& \mathbf{H}(z)=\left[\mathbf{I}_{m}+\mathbf{G}(z) \mathbf{K}(z)\right]^{-1} \mathbf{G}(z) \mathbf{K}(z) \\
& \mathbf{G}(z)=\left(z \mathbf{I}_{m}-\mathbf{I}_{m}+\hat{\mathbf{B}}_{0}^{-1} \hat{\mathbf{B}}_{1}\right)^{-1} \hat{\mathbf{B}}_{0}^{-1}
\end{aligned}
$$

The controller transfer function matrix $\mathbf{K}(z)$ here is assumed to be generated by a one-dimensional discrete linear system with state-space quadruple $\{\mathbf{P}, \mathbf{M}, \mathbf{N}, \mathbf{Q}\}$.

Suppose now that, with equation (34) in place, $\mathbf{K}(z)$ is chosen as proportional control applied on the current pass. Then the general parametric form of this controller is (by analogy with the one-dimensional case)

$$
\mathbf{K}(z)=\hat{\mathbf{B}}_{0} \operatorname{diag}\left(1-k_{j}\right)_{1 \leqslant j \leqslant m}-\hat{\mathbf{B}}_{1}
$$

Also, introduce $\rho_{0}(z)=\operatorname{det}\left(z \mathbf{I}_{m}-\mathbf{\Phi}\right)$ which in this particular case is given by

$$
\rho_{0}(z) \equiv \operatorname{det}\left(\hat{\mathbf{B}}_{0}^{-1}\right) \operatorname{det}\left(\mathbf{G}^{-1}(z)\right)
$$

where $\mathbf{G}^{-1}(z)$ is defined by (30). Hence, since asymptotic stability holds both open and closed loop, it follows immediately that closed-loop stability along the pass holds if, and only if,

$$
\begin{aligned}
\rho_{\mathrm{c}}(z) & =\rho_{0}(z) \operatorname{det}\left[\mathbf{I}_{m}+\mathbf{G}(z) \mathbf{K}(z)\right] \\
& =\operatorname{det}\left(\hat{\mathbf{B}}_{0}^{-1}\right) \operatorname{det}\left[\mathbf{G}^{-1}(z)+\mathbf{K}(z)\right] \\
& =\prod_{j=1}^{m}\left(z-k_{j}\right) \neq 0, \quad \forall|z| \geqslant 1
\end{aligned}
$$

and hence, closed-loop stability along the pass if, and only if, $k_{j} \in(-1,1), 1 \leqslant j \leqslant m$.

Under this form of control scheme, the limit profile is reached on the first pass and its dynamics is defined by the transfer function $\mathbf{H}(z)$. As in the one-dimensional linear systems case, closed-loop transient performance can be assessed by evaluation of the closed-loop transfer function matrix, i.e.

$$
\begin{aligned}
\mathbf{H}(z) & =\left[\mathbf{I}_{m}+\mathbf{G}(z) \mathbf{K}(z)\right]^{-1} \mathbf{G}(z) \mathbf{K}(z) \\
& =\left[\mathbf{G}^{-1}(z)+\mathbf{K}(z)\right]^{-1} \mathbf{K}(z) \\
& =\operatorname{diag}\left(\frac{1}{z-k_{j}}\right)_{1 \leqslant j \leqslant m}\left[\operatorname{diag}\left(1-k_{j}\right)_{1 \leqslant j \leqslant m}-\hat{\mathbf{B}}_{0}^{-1} \hat{\mathbf{B}}_{1}\right]
\end{aligned}
$$

Hence, the closed-loop limit profile will have small steady state errors and small interaction effects in response to unit step commands only provided the elements of the matrix $\hat{\mathbf{B}}_{0}^{-1} \hat{\mathbf{B}}_{1}$ are 'sufficiently small'. In particular, it will have these properties if the sampling rate is 'fast enough'.

To discuss this last point further, suppose (for simplicity) that matrix $\mathbf{A}$ of the underlying differential process has a diagonal canonical form with eigenvalues $\lambda_{j}$, $1 \leqslant j \leqslant m$, and eigenvector matrix $\mathbf{E}$. Then

$$
\boldsymbol{\Phi}=\mathbf{E} \operatorname{diag}\left(\mathrm{e}^{\lambda_{i} h}\right)_{1 \leqslant j \leqslant m} \mathbf{E}^{-1}
$$

suggests that a necessary condition for $\hat{\mathbf{B}}_{0}^{-1} \hat{\mathbf{B}}_{1}$ to be 'small' is that

$$
\left|\lambda_{j} h\right| \ll 1, \quad 1 \leqslant j \leqslant m
$$

Equivalently, the sampling rate must be 'fast' in comparison with the eigenvalues of matrix $\mathbf{A}$ in the underlying differential process.

The above analysis is easily extended to the choice of $\mathbf{K}(z)$ as a controllable and observable proportional-plussummation controller of the general parametric form 


$$
\begin{aligned}
\mathbf{K}(z)= & \hat{\mathbf{B}}_{0} \operatorname{diag}\left[1-k_{j} c_{j}+\frac{\left(1-k_{j}\right)\left(1-c_{j}\right)}{z-1}\right]_{1 \leqslant j \leqslant m} \\
& -\hat{\mathbf{B}}_{1}
\end{aligned}
$$

Suppose that only $q$ of the parameters $c_{j}, 1 \leqslant j \leqslant m$, are not equal to unity. Then it follows immediately that the controller $(\mathbf{K}(z))$ has minimal realization of state dimension $q$, and hence that in this case

$$
\begin{aligned}
\rho_{0}(z) & =(z-1)^{q} \operatorname{det}\left(z \mathbf{I}_{m}-\boldsymbol{\Phi}\right) \\
& =(z-1)^{q} \operatorname{det}\left(\hat{\mathbf{B}}_{0}^{-1}\right) \operatorname{det}\left[\mathbf{G}^{-1}(z)\right]
\end{aligned}
$$

Note now that the analysis reduces to the previous case (proportional action only) if $q=0$, and that closed-loop stability along the pass here holds if, and only if,

$$
\begin{aligned}
\rho_{\mathrm{c}}(z) & =\rho_{0}(z) \operatorname{det}\left[\mathbf{I}_{m}+\mathbf{G}(z) \mathbf{K}(z)\right] \\
& =(z-1)^{q} \operatorname{det}\left(\hat{\mathbf{B}}_{0}^{-1}\right) \operatorname{det}\left(\mathbf{G}^{-1}(z)+\mathbf{K}(z)\right) \\
& =\left[\prod_{j=1}^{m}\left(z-k_{j}\right)\right]\left[\prod_{c_{j} \neq 1}\left(z-c_{j}\right)\right]
\end{aligned}
$$

i.e. closed-loop stability along the pass if, and only if, $k_{j} \in(-1,1)$ and $c_{j} \in(-1,1], 1 \leqslant j \leqslant m$.

In practice, the inclusion of summation action would normally be considered in cases where proportional action displayed large steady state errors. Also, the reset times will normally be considerably longer than the rise times, i.e. it is likely that

$$
\left|1-c_{j}\right| \ll\left|1-k_{j}\right|, \quad 1 \leqslant j \leqslant m
$$

and hence $\mathbf{H}(z)$ of (40) is still a good representation of the closed-loop limit profile dynamics, with the exception that steady state errors in response to step commands will be zero in all channels of the limit profile output vector $\boldsymbol{y}_{\infty}(p)$ corresponding to parameters $c_{j} \neq 1$. This fact is easily verified by noting that

$$
\begin{aligned}
\lim _{z \rightarrow 1} \mathbf{H}(z) & =\lim _{z \rightarrow 1}\left[\mathbf{G}^{-1}(z)+\mathbf{K}(z)\right]^{-1} \mathbf{K}(z) \\
& =\mathbf{I}_{m}-\mathbf{M} \hat{\mathbf{B}}_{0}^{-1} \hat{\mathbf{B}}_{1}
\end{aligned}
$$

where

$$
\mathbf{M}=\operatorname{diag}\left(m_{j}\right)_{1 \leqslant j \leqslant m}
$$

and

$$
m_{j}= \begin{cases}0, & c_{j} \neq 1 \\ \frac{1}{1-k_{j}}, & c_{j}=1\end{cases}
$$

The analysis just completed is, in effect, a rather idealized concept of control action and, in practice, there may be significant delays in the control loops owing, for example, to measurement or actuator delays in the system, where here it is assumed that these occur on the current pass. Essentially, these delays will not adversely affect system performance provided that the sampling rate is 'fast' compared with the (current pass) closedloop system responses.
This last point can be illustrated by considering the presence of current pass measurement delays represented by the simple diagonal matrix

$$
\mathbf{F}(z)=z^{-d} \mathbf{I}_{m}, \quad d \geqslant 0
$$

in the feedback path. Considering (for simplicity) the proportional version of $\mathbf{K}(z)$ defined by (37) gives

$$
\begin{aligned}
& \rho_{\mathrm{c}}(z)= z^{m d} \operatorname{det}\left(\hat{\mathbf{B}}_{0}^{-1}\right) \operatorname{det}\left[\mathbf{G}^{-1}(z)\right] \operatorname{det}\left[\mathbf{I}_{m}+\mathbf{G}(z) \mathbf{K}(z) \mathbf{F}(z)\right] \\
&=z^{m d} \operatorname{det}\left(\hat{\mathbf{B}}_{0}^{-1}\right) \operatorname{det}\left[\mathbf{G}^{-1}(z)+\mathbf{K}(z) \mathbf{F}(z)\right] \\
&=z^{m d} \operatorname{det}\left\{(z-1) \mathbf{I}_{m}+\hat{\mathbf{B}}_{0}^{-1} \hat{\mathbf{B}}_{1}\right. \\
&\left.\quad \quad+z^{-d}\left[\operatorname{diag}\left(1-k_{j}\right)_{1 \leqslant j \leqslant m}-\hat{\mathbf{B}}_{0}^{-1} \hat{\mathbf{B}}_{1}\right]\right\} \\
& \quad \operatorname{det}\left\{\operatorname{diag}\left[z^{d}(z-1)+1-k_{j}\right]_{1 \leqslant j \leqslant m}\right. \\
&\left.\quad+\left(z^{d}-1\right) \hat{\mathbf{B}}_{0}^{-1} \hat{\mathbf{B}}_{1}\right\}
\end{aligned}
$$

Using this last equation, it follows that at all 'high enough' sampling rates the term $\hat{\mathbf{B}}_{0}^{-1} \hat{\mathbf{B}}_{1}$ can be neglected to yield as $h \rightarrow 0$

$$
\rho_{\mathrm{c}}(z) \approx \prod_{j=1}^{m}\left[z^{d}(z-1)+\left(1-k_{j}\right)\right]
$$

Considering solutions of $z^{d}(z-1)+1-k_{j}=0$ in the vicinity of $z=k_{j}=1$, the relation

$$
\left.\frac{\mathrm{d} z}{\mathrm{~d} k_{j}}\right|_{z=k_{j}=1}=1
$$

guarantees the existence of positive real numbers $\varepsilon_{j}>0$, $1 \leqslant j \leqslant m$, such that the solutions of $\rho_{\mathrm{c}}(z)=0$ lie in the open unit circle in the complex plane for $1-\varepsilon_{j}<k_{j}<1$, $1 \leqslant j \leqslant m$, and all 'high enough' sampling rates.

\section{AN EXTENSION}

The analysis of the first part of this section makes use of the following result for one-dimensional discrete-time linear systems that was originally given in reference [8] [where $\mathbf{Q}(z)$ is the forward path transfer function of the unity negative feedback control scheme with plant transfer function $\mathbf{G}(z)$ and forward path transfer function matrix $\mathbf{K}(z)$, i.e. $\mathbf{Q}(z)=\mathbf{G}(z) \mathbf{K}(z)]$.

\section{Theorem 3}

Suppose that the controllable and observable onedimensional minimum phase $m \times m$ discrete-time linear system $\mathbf{Q}(z)$ is to be approximated by the invertible minimum phase one-dimensional discrete-time linear system $\mathbf{Q}_{\mathbf{A}}(z)$. Suppose also that $\mathbf{Q}_{\mathrm{A}}(z)$ is stable under unity negative feedback control and that the poles of the closed-loop system generated by $\mathbf{Q}(z)$ under unity negative feedback control lie in the open ball $|z|<\mathbf{R}$, where $\mathbf{R}>1$. Then the system $\mathbf{Q}(z)$ is stable under unity negative feedback control if the following so-called contraction condition holds:

$$
\left\|\left(\mathbf{I}_{m}+\mathbf{Q}_{\mathrm{A}}^{-1}(z)\right)^{-1}\left(\mathbf{Q}_{\mathrm{A}}^{-1}(z)-\mathbf{Q}^{-1}(z)\right)\right\|<1
$$


where, if $\mathbf{E}(z)$ is any $m \times m$ matrix function of $z$,

$$
\|\mathbf{E}(z)\|:=\max _{1 \leqslant j \leqslant m} \max _{|z|=1,|z|=\mathbf{R}} \sum_{i=1}^{m}\left|\mathbf{L}_{j i}(z)\right|
$$

This result is proved by noting that the controllability and observability assumptions ensure that the closedloop system is stable in the one-dimensional asymptotic sense if, and only if, the matrix

$$
\left(\mathbf{I}_{m}+Q(z)\right)^{-1} \mathbf{Q}(z) \equiv\left(\mathbf{I}_{m}+\mathbf{Q}^{-1}(z)\right)^{-1}
$$

is analytic and bounded in the region $1<|z|<\mathbf{R}$, i.e. if, and only if, the solution of

$$
y(z)=-\mathbf{Q}^{-1}(z) \boldsymbol{y}(z)+\beta
$$

is analytic and bounded in $1<|z|<\mathbf{R}$ independently of the choice of $\beta \in \mathbb{R}^{m}$.

Now write equation (57) in the form

$$
\boldsymbol{y}(z)=\left[\mathbf{I}_{m}+\mathbf{Q}_{\mathrm{A}}^{-1}(z)\right]^{-1}\left\{\left[\mathbf{Q}_{\mathrm{A}}^{-1}(z)-\mathbf{Q}^{-1}(z)\right] \boldsymbol{y}(z)+\beta\right\}
$$

then the remainder of the proof is identical to that referenced in reference [8] for the equivalent one-dimensional differential linear systems case with the Nyquist contour replaced by the closed region $1 \leqslant|z| \leqslant \mathbf{R}$. Hence, the details are omitted here.

In practice, $\mathbf{R}$ will, of course, be unknown, but this does not limit the applicability of the result since $\mathbf{R} \gg 1$ can always be taken. Given $\mathbf{Q}(z)$ and $\mathbf{Q}_{\mathrm{A}}(z)$, the contraction condition (55) can be tested numerically but its major use here is for theoretical closed-loop stability analysis of the previous section to the control of discrete linear repetitive processes.

Return, therefore, to the analysis of the previous section and suppose that the inverse of the transfer function matrix $\mathbf{G}(z)$ is of the form

$$
\mathbf{G}^{-1}(z)=(z-1) \hat{\mathbf{B}}_{0}+\hat{\mathbf{B}}_{1}+\hat{\mathbf{B}}_{0} \hat{\mathbf{H}}(z)
$$

where $\hat{\mathbf{H}}(z)$ is proper with $\hat{\mathbf{H}}(1)=0$ and, as before, $\operatorname{det}\left(\hat{\mathbf{B}}_{0}\right) \neq 0$. Also the $m \times m$ multivariable first-order lag transfer function matrix $\mathbf{G}_{\mathbf{a}}(z)$ defined as

$$
\mathbf{G}_{\mathrm{a}}^{-1}(z)=(z-1) \hat{\mathbf{B}}_{0}+\hat{\mathbf{B}}_{1}
$$

is to be used as a reduced-order model for controller design purposes. Note that $\mathbf{G}_{\mathbf{a}}(z)$ is a good approximation to the high-frequency and steady state behaviour of the one-dimensional linear system defined by the statespace triple $\{\boldsymbol{\Phi}, \boldsymbol{\Delta}, \mathbf{C}\}$ but contains no information on its zero structure - in fact, $\mathbf{G}_{\mathrm{a}}(z)$ has no zeros.

Suppose now that the analysis of the previous section is used to design a proportional-plus-summation controller of form (43) for unity negative feedback control of the one-dimensional discrete linear system described by $\mathbf{G}_{\mathrm{a}}(z)$. Suppose also that this controller is used in repetitive process control law (33) with $\mathbf{K}_{1}$ again defined by equation (34). Then, contraction condition (55) can be used to assess closed-loop stability along the pass of the resulting discrete linear repetitive process. The relevant matrix is

$$
\begin{aligned}
\mathbf{E}(z) & =\left(\mathbf{I}_{m}+\mathbf{Q}_{\mathrm{a}}^{-1}(z)\right)^{-1}\left(\mathbf{Q}_{\mathrm{a}}^{-1}(z)-\mathbf{Q}^{-1}(z)\right) \\
& =\left(\mathbf{K}(z)+\mathbf{G}_{\mathrm{a}}^{-1}(z)\right)^{-1}\left(\mathbf{G}_{\mathrm{a}}^{-1}(z)-\mathbf{G}^{-1}(z)\right) \\
& =(-1) \operatorname{diag}\left[\frac{z-1}{\left(z-k_{j}\right)\left(z-c_{j}\right)}\right]_{1 \leqslant j \leqslant m} \mathbf{H}(z)
\end{aligned}
$$

and a sufficient condition for closed-loop stability along the pass is that $\|\mathbf{E}(z)\|<1$.

It would be possible directly to investigate this last condition if the inverse system were computed from the state-space triple $\{\boldsymbol{\Phi}, \boldsymbol{\Delta}, \mathbf{C}\}$ using known techniques (see, for example, reference [9]) and noting the fact that the condition $\operatorname{det}\left(\hat{\mathbf{B}}_{0}\right) \neq 0$ is equivalent to the condition $\operatorname{det}(\mathbf{C} \boldsymbol{\Delta}) \neq 0$. Note also that the contraction condition here can be replaced by the simpler condition

$$
\max _{1 \leqslant j \leqslant m} \max _{|z|=1} \sum_{j=1}^{m}\left|\mathbf{E}_{j i}(z)\right|<1
$$

by letting $\mathbf{R} \rightarrow \infty$ and noting that $\mathbf{E}(z)=\mathbf{O}\left(z^{-1}\right)$.

The question that must now be addressed is whether or not the contraction condition can be satisfied for a 'reasonable' class of examples. In which context, suppose that discrete linear repetitive process (11) can be regarded as a discrete representation of differential linear repetititve process (1) with sampling period $h>0$. Then the following result will be obtained.

\section{Theorem 4}

Suppose that the one-dimensional differential linear time-invariant system $S(\mathbf{A}, \mathbf{B}, \mathbf{C})$ formed from the differential linear repetitive process state-space model (1) is minimum phase and $\operatorname{det}(\mathbf{C B}) \neq 0$. Suppose also that this repetitive process is to be controlled by a control law of the form (33) and (34), where $\hat{\boldsymbol{y}}_{k^{+}}{ }_{1}(p)$ is generated by a $\mathbf{K}(z)$ of form (43). Then, for each choice of parameters $k_{j}$ and $c_{j}, 1 \leqslant j \leqslant m$, there exists a positive real number $h^{*}$ such that for $h \in\left(0, h^{*}\right)$ the onedimensional discrete linear time-invariant system $S(\boldsymbol{\Phi}, \Delta, \mathbf{C})$ of the resulting discrete linear repetitive process is minimum phase with the inverse of the form given by equation (59), and the contraction condition for closed-loop stability along the pass holds.

Proof. Firstly note that $S(\boldsymbol{\Phi}, \boldsymbol{\Delta}, \mathbf{C})$ is the inverse of the form given in equation (59) if, and only if, $\mathbf{C} \boldsymbol{\Delta}\left(=\hat{\mathbf{B}}_{0}^{-1}\right)$ is non-singular. Using (23),

$$
\lim _{h \rightarrow 0} h^{-1} \mathbf{C} \boldsymbol{\Delta}=\mathbf{C B}
$$

which, by assumption, is non-singular. It therefore follows that equation (59) is the correct parametric representation of the inverse system at all high enough sampling rates.

Using results in reference [9], note that

$$
\mathbf{G}^{-1}(z)
$$




$$
\begin{aligned}
= & (z-1)(\mathbf{C} \boldsymbol{\Delta})^{-1}-(\mathbf{C} \boldsymbol{\Delta})^{-1} \mathbf{C}\left\{\boldsymbol{\Phi}-\mathbf{I}_{n}\right\} \boldsymbol{\Delta}(\mathbf{C} \boldsymbol{\Delta})^{-1} \\
& -(\mathbf{C} \boldsymbol{\Delta})^{-1} \mathbf{C}\left\{\boldsymbol{\Phi}-\mathbf{I}_{n}\right\} \mathbf{M}\left(z \mathbf{I}_{n}-\mathbf{N} \boldsymbol{\Phi} \mathbf{M}\right)^{-1} \\
& \times \mathbf{N}\left\{\boldsymbol{\Phi}-\mathbf{I}_{n}\right\} \boldsymbol{\Delta}(\mathbf{C} \boldsymbol{\Delta})^{-1}
\end{aligned}
$$

where $\mathbf{N}$ and $\mathbf{M}$ are $(n-m) \times n$ and $n \times(n-m)$ full-rank matrices respectively, which satisfy

$$
\mathbf{C M}=0, \quad \mathbf{N} \boldsymbol{\Delta}=0, \quad \mathbf{N M}=\mathbf{I}_{n-m}
$$

It follows immediately that

$$
\begin{aligned}
\mathbf{H}(z)= & \mathbf{C}\left\{\boldsymbol{\Phi}-\mathbf{I}_{n}\right\} \\
& \times \mathbf{M}\left[\left\{\mathbf{I}_{n}-\mathbf{N} \boldsymbol{\Phi} M\right\}^{-1}-\left(z \mathbf{I}_{n}-\mathbf{N} \boldsymbol{\Phi} \mathbf{M}\right\}^{-1}\right] \\
& \times \mathbf{N}\left\{\boldsymbol{\Phi}-\mathbf{I}_{n}\right\} \Delta(\mathbf{C} \Delta)^{-1} \\
= & \mathbf{C}\left\{\boldsymbol{\Phi}-\mathbf{I}_{n}\right\} \mathbf{M}(z-1)\left(\mathbf{I}_{n}-\mathbf{N} \boldsymbol{\Phi} \mathbf{M}\right)^{-1} \\
& \times\left(z \mathbf{I}_{n}-\mathbf{N} \boldsymbol{\Phi} \mathbf{M}\right)^{-1} \mathbf{N}\left\{\boldsymbol{\Phi}-\mathbf{I}_{n}\right\} \Delta(\mathbf{C} \Delta)^{-1}
\end{aligned}
$$

Now consider the behaviour of $\mathbf{H}(z)$ as $h \rightarrow 0$ on the unit circle $|z|=1$. Then the eigenvalues $\lambda_{1}, \ldots, \lambda_{n^{-} m}$ of $\mathbf{N \Phi M}$ are simply the $n-m$ zeros [9] of $S(\boldsymbol{\Phi}, \boldsymbol{\Delta}, \mathbf{C})$, i.e. the $n-m$ solutions of

$$
z(\lambda)=\operatorname{det}\left(\begin{array}{cc}
\lambda \mathbf{I}_{n}-\boldsymbol{\Phi} & -\boldsymbol{\Delta} \\
\mathbf{C} & 0
\end{array}\right)=0
$$

Then, since

$$
\begin{aligned}
\boldsymbol{\Phi} & =\mathbf{I}_{n}+\mathbf{A} h+\mathbf{O}\left(h^{2}\right) \\
\boldsymbol{\Delta} & =h \mathbf{B}+\mathbf{O}\left(h^{2}\right)
\end{aligned}
$$

it follows that

$$
\lambda_{j}=1+z_{j} h+\mathbf{O}\left(h^{2}\right), \quad 1 \leqslant j \leqslant n-m
$$

where $z_{1}, \ldots, z_{n^{-} m}$ are the zeros of the continuous time system $S(\mathbf{A}, \mathbf{B}, \mathbf{C})$. By the minimum phase assumption, all of these numbers have strictly negative real parts, and hence $\left\{\lambda_{j}\right\}_{1 \leqslant j \leqslant n^{-} m}$ lie in the open unit circle in the complex plane for all 'fast enough' sampling rates. Equivalently, $S(\boldsymbol{\Phi}, \boldsymbol{\Delta}, \mathbf{C})$ is minimum phase for all 'fast enough' sampling rates.

To prove the last part, suppose, without loss of generality, that $\mathbf{M}$ is constant. Then the assumption that $\operatorname{det}(\mathbf{C B}) \neq 0$ ensures that the limit $\mathbf{N}_{0}:=\lim _{h \rightarrow 0} \mathbf{N}$ exists, and also that $\mathbf{N}_{0} \mathbf{M}=\mathbf{I}_{n-m}$ and $\mathbf{N}_{0} \mathbf{B}=0$. Also, the minimum phase nature of $S(\mathbf{A}, \mathbf{B}, \mathbf{C})$ ensures that $\operatorname{det}\left(\mathbf{N}_{0} \mathbf{A M}\right) \neq 0$, and this fact, together with (63) and (68) and the identity

$$
\left(z \mathbf{I}_{n}-\mathbf{N} \boldsymbol{\Phi} \mathbf{M}\right)^{-1}=\left[(z-1) \mathbf{I}_{n}-\mathbf{N}_{0} \mathbf{A M} h+\mathbf{O}\left(h^{2}\right)\right]^{-1}
$$

guarantees that there exists $h^{*}>0$ and $\boldsymbol{e}>0$ such that

$$
\sup _{0<h^{<} h^{*}}\left\|(z-1)^{-1} \mathbf{H}(z)\right\|<\boldsymbol{e}
$$

In particular, $(z-1)^{-1} \mathbf{H}(z)$ converges uniformly to zero as $h \rightarrow 0$ on any relatively open subset $T$ of the unit circle not containing the point $z=1$ from which $\mathbf{H}(z)$ [and hence $\mathbf{E}(z)$ ] converges uniformly to zero on $|z|=1$ as $h \rightarrow 0$. This completes the proof of the result since suitable choice of $h^{*}$ ensures that $\|\mathbf{E}(z)\|<1,0<h<h^{*}$.

In effect, this last theorem states that the contraction condition for closed-loop stability along the pass is always satisfied if the one-dimensional differential system $S(\mathbf{A}, \mathbf{B}, \mathbf{C})$, which, in effect, describes the contribution of the current pass input vector to the pass profile of a differential linear repetitive process with state-space model (1), is minimum phase, uniform rank one, and the sampling rate is fast enough. Note also that these conditions are sufficient only and do not necessarily prevent application of the technique if they do not hold.

The following is a systematic procedure for the application of Theorem 4:

Step 1. Evaluate the matrices $\hat{\mathbf{B}}_{0}$ and $\hat{\mathbf{B}}_{1}$. One way of doing this is from the matrices $\boldsymbol{\Phi}, \boldsymbol{\Delta}, \mathbf{C}$, using known formulae for the inverse of one-dimensional discrete linear systems (see, for example, reference [9] and the relevant cited references). Alternatively, if $r(\boldsymbol{\Phi})<1$, these matrices may be deduced by obtaining the response of the one-dimensional discrete linear system $S(\boldsymbol{\Phi}, \boldsymbol{\Delta}, \mathbf{C})$ to step inputs from zero initial conditions. In particular, the output responses of this system from zero initial conditions to the vector step demand $\boldsymbol{r}$ at $p=1$ and $p=\infty$ are

$$
\boldsymbol{y}_{1}=\hat{\mathbf{B}}_{0}^{-1} \boldsymbol{r}, \quad \boldsymbol{y}_{\infty}=\hat{\mathbf{B}}_{1}^{-1} \boldsymbol{r}
$$

and $\hat{\mathbf{B}}_{0}$ and $\hat{\mathbf{B}}_{1}$ are easily evaluated from these data by taking $m$ linearly independent step demands $r_{1}, r_{2}, \ldots, r_{m}$.

Step 2. Compute $\mathbf{K}_{1}$ of (34) and choose a proportionalplus-summation controller of form (43) to meet the performance specifications on the resulting closedloop limit profile.

Step 3. Check stability along the pass of the actual closed-loop system by a numerical check of contraction condition (55). If the system is unstable along the pass, consider the possibility of increasing the sampling rate.

The above theory will remain essentially the same in the presence of sensor or actuator delays, provided that the sampling rate is fast enough (relative to the designed closed-loop limit profile responses), and also that the requirement that $\hat{\mathbf{H}}(1)=0$ in equation (59) can be removed without altering the results. To see that this is indeed possible, firstly note that $\hat{\mathbf{B}}_{1}$ represents steady state response characteristics and hence it is independent of the sampling rate. Suppose also that $\tilde{\mathbf{B}}_{1}$ is some other constant $m \times m$ matrix that is used instead of $\hat{\mathbf{B}}_{1}$. Then, since the theoretical development is, with the exception of Theorem 4 , independent 
of the assumption $\hat{\mathbf{H}}(1)=0$, the analysis follows through with $\hat{\mathbf{H}}(z)$ replaced by $\mathbf{H}(z)+\hat{\mathbf{B}}_{0}^{-1}\left(\hat{\mathbf{B}}_{1}-\tilde{\mathbf{B}}_{1}\right)$. Also, $\mathbf{B}_{0}^{-1}=\mathbf{C} \boldsymbol{\Delta}=\mathbf{O}(h)$ yields

$$
\lim _{h \rightarrow 0} \hat{\mathbf{B}}_{0}^{-1}\left(\hat{\mathbf{B}}_{1}-\tilde{\mathbf{B}}_{1}\right)=0
$$

and it follows immediately that Theorem 4 is valid if $\hat{\mathbf{B}}_{1}$ is replaced with any constant matrix.

\section{CONCLUSIONS}

This paper has developed an approach to controller design for the class of two-dimensional systems known as differential linear repetitive processes. This is based on fast sampling control using a control law that is a combination of one-dimensional error-actuated output feedback control on the current pass augmented by feedforward proportional control action from the previous pass which cancels the effects of the previous pass term from the resulting closed-loop system. These results provide a benchmark for future research on an important class of systems for which, until recently, most of the control-related analysis has focused on systems theoretic aspects such as stability theory and controllability and observability analysis.

\section{REFERENCES}

1 Edwards, J. B. Stability problems in the control of multipass processes. Proc. Instn Elect. Engrs, 1974, 121, 1425-1431.

2 Smyth, K. J. Computer aided analysis for linear repetitive processes. PhD thesis, University of Strathclyde, Glasgow, 1992.

3 Amann, N., Owens, D. H. and Rogers, E. Predictive optimal iterative learning control. Int. J. Control, 1998, 69(2), 203-226.

4 Roberts, P. D. Stability analysis of iterative optimal control algorithms modelled as linear repetitive processes. Proc. Instn Elect. Engrs, Control Theory and Applic., Part D, 2000, 147(3), 229-238.

5 Rogers, E. and Owens, D. H. Stability Analysis for Linear Repetitive Processes. Lecture Notes in Control and Information Sciences Series, 1992, Vol.175 (SpringerVerlag, Berlin).

6 Rogers, E., Galkowski, K. and Owens, D. H. Control Systems Theory and Applications for Linear Repetitive Processes. Lecture Notes in Control and Information Sciences Series, 2002 (Springer-Verlag, Berlin) (to appear).

7 Owens, D. H. and Rogers, E. Stability analysis for a class of 2D continuous-discrete linear systems with dynamic boundary conditions. Syst. and Control Lett., 1999, 37, 55-60.

8 Owens, D. H. Discrete first-order models for multivariate process control. Proc. Instn Elect. Engrs, Control Theory and Applic., Part D, 1979, 126(11), 525-530.

9 Kouvaritakis, B. A geometric approach to the inversion of multivariate systems. Int. J. Control, 1976, 24, 609-626. 